\title{
Detection of Gastric Cancer at Early Stage is More Effective than we Consider? A New Phenomenon that can be Taken into Consideration
}

\author{
Ramiz Bayramov*, Ramila T Abdullayeva and Simare E Huseynova \\ Department of Oncology, Azerbaijan Medical University, Baku, Azerbaijan \\ *Corresponding Author: Ramiz Bayramov, Department of Oncology, Azerbaijan \\ Medical University, Baku, Azerbaijan.
}

Received: May 28, 2020

Published: June 30, 2020

(C) All rights are reserved by Ramiz

Bayramov., et al.
Despite the fact that the incidence and mortality of gastric cancer have fallen dramatically over the past several decades it remains a major public health issue as the fifth most common cancer (excluding skin cancer) and the second leading cause of cancer death worldwide. Despite its decreasing trend gastric cancer's incidence, however, continues to be the highest among all cancers in some countries. The epidemiology of gastric cancer has substantial geographical heterogeneity, and its incidence can vary 10 -fold between high-risk and low-risk countries. Also, geographic variability in prognosis for gastric cancer is well documented. So, the 5-year survival rate is $65-70 \%$ in South Korea and Japan while it is around $20-30 \%$ in the other countries. In the Eastern series (primarily from South Korea and Japan) higher survival rates were reported when compared to Western patients at the same stage of the disease following the same surgery. The highest survival rate in the mentioned countries mainly is attributed to population screening that has led to the diagnosis of cancer at early stages in most of the cases. Epidemiological studies revealed linear correlation between the incidence of gastric cancer and relative survival but real impact of this factor on prognosis remains uncertain. Even molecular studies conducted on Eastern and Western patients revealed no potential factors that could impact on differences in survival.

As mentioned above differences in survival rates were also observed between Eastern and Western patients underwent the same radical intent surgery for gastric cancer at the same stage of the disease. Some explanations have been pronounced over these differences including "stage migration phenomenon" related to the differences in BMI of the appropriate patients and consequently to the level of adequacy of lymph node dissection, possible diversity in biological properties of cancer cells etc. We suggest that there is another phenomenon that can impact on the survival rate of gastric cancer patients in any given region that we called "effect of the proportion of early gastric cancer". The phenomenon means that the highest is the proportion of early gastric cancer (EGC) the highest is the survival rate after radical intent surgery for any resectable stage of gastric cancer, just not for EGC. How potential can be this effect? How can it be explained?

First of all when we conducted meta-analysis of available to as studies it was observed that proportion of EGC affects reversely on the proportion of $\mathrm{N}+$ cases in patients with gastric cancer. But in those articles we did not find stratification of $\mathrm{N}$ stages according to $\mathrm{T}$ stages. When we retrospectively compared proportion of EGC, proportion of N0 cases among T2-T4a tumors and the survival rates of the patients with $\mathrm{T} 2-\mathrm{T} 4 \mathrm{a}$ gastric cancer after radical intent surgery in different time periods we observed that as the proportion of EGC increases in parallel increases also the proportion of N0 cases among T2-T4a tumors and the 5-year survival rate (R.B. Bayramov, R.T. Abdullayeva. Europ J Surg Oncol, 2014; 11: S22-S23). In this context study of Marrelli D., et al. (Br J Surg, 2011; 98 (9): 273-1283) deserves a thorough analysis. The researchers analyzed surgical, pathological and follow-up data of 2822 patients who underwent radical intent surgery in 1991 - 2005 years using the prospective centralized database. The analysis focused on three periods: 1991 - 1995 (period 1 - 1024 pts), 1996 - 2000 (period 2 - 955 pts) and 2001 - 2005 (period 3 - 843 pts). Despite the fact that in $63.8 \%$ of the patients from period 1 and $89.5 \%$ from period 3 were harvested $\geq 15$ lymph nodes 5 -year survival rate for pT2 and for pT3-4 tumor was $73.9 \%$ vs $65.3 \%$ ( $p=0.131$ ) and $42.1 \%$ vs $28.1 \%$ ( $p=0.031$ ), respectively. The authors state that "...the observed changes in clinical and pathological features cannot completely explain the worse outcome, as treatment period was identified as an independent negative prognostic factor in the multivariable analysis. Other time-related unknown clinical or biological factors may be involved". When analyzing the pathological data of the paper we observed that patients with T1 stage constituted $29.2 \%$ of all the patients from period 1 and $23.8 \%$ - from period $3(p<0.001)$, that was not taken into consideration properly by authors.

We tried to give an explanation for those paradoxical results according to the aforementioned phenomenon - "effect of the proportion of early gastric cancer" suggested by us. Theoretically every resectable stage (I-III stages) of cancer can be subdivided into two subcategories - without and with synchronous occult (microscopic) distant metastases. Unfortunately, today we are unable to establish the existence of distant micrometastases. That is why we categorize both "subcategories" in one stage but really they are distinct "stages". It is known that some proportion of patients with gastric cancer of any resectable stage is cured and the another part develops metachronous obvious distant metastases in some period of time following radical intent surgery. It is undeniable fact that most of the cured patients are those who had no synchronous 
occult distant metastases before radical intent surgery. It should be noted that the development of hematogenous dissemination is time-dependent process. So, by means of screening not only more proportion of patients can be detected at T1 (EGC) stage, accordingly more proportion of patients at > T1 stage can be caught without synchronous occult distant metastases as well. In other words more proportion of EGC can be indirectly and in parallel associated with more proportion of $>\mathrm{T} 1$ stage-gastric cancer cases without synchronous occult distant metastases. According to this logic more proportion of EGC can be detected without lymphogenic metastases that can be positively reflected in the long-term results of endoscopic mucosectomy in countries, where the proportion of EGC is higher. Therefore, the higher proportion of EGC can be a positive determinant of prognosis for gastric cancer of any resectable stage in any given large cohort of patients.

In summery the suggested phenomenon can be one of the reasonable explanations why higher survival rates after radical intent surgery for gastric cancer have been observed in areas with higher incidence of gastric cancer (in South Korea and Japan) when compared to the areas with lower incidence at the same resectable stage of the disease after the same surgery. As explained above it can be due to detection of more proportion of patients with gastric cancer at EGC stage in South Korea and Japan compared to Western countries (40 - $60 \%$ vs 10 - 20\%, respectively).

It is important to note that as the proportion of EGC increases, inversely decreases the proportion of the patients with IV stagegastric cancer. That is why to consider that the proportion of IV stage-gastric cancer also can be a determinant of prognosis for any resectable gastric cancer in a large cohort of patients is not beyond logic. On the other hand today's obvious hematogenous metastasis was occult metastasis certain time ago. Theoretically it means that the most is the proportion of IV stage-gastric cancer the most is the proportion of resectable stage-gastric cancer with occult metastases (micrometastases) in any given large cohorts of the patient.

The phenomenon can generate some hypotheses that need to be studied in a large cohort of patients:

1. In regions with lower proportion of EGC adjuvant chemotherapy might demonstrate more effectiveness compared to the regions with higher proportion of EGC due to more percentage of cases with possible synchronous occult distant metastases;

2. In regions with highest proportion of EGC endoscopic mucosectomy can be performed more frequently without fear of recurrences in regional lymph nodes;

3. The proportion of IV stage-gastric cancer in a large cohort of patients can predict the approximate prognosis for gastric cancer of any resectable stage after radical intent surgery;

4. This phenomenon can concern not only gastric cancer also other cancers with high incidence rate.

\section{Assets from publication with us}

- Prompt Acknowledgement after receiving the article

- Thorough Double blinded peer review

- Rapid Publication

- Issue of Publication Certificate

- High visibility of your Published work

Website: https://www.actascientific.com/

Submit Article: https://www.actascientific.com/submission.php

Email us: editor@actascientific.com

Contact us: +919182824667 